Check for updates

Cite this: Chem. Sci., 2018, 9, 8071

๑ All publication charges for this article have been paid for by the Royal Society of Chemistry

\title{
A miniaturized solid salt reverse electrodialysis battery: a durable and fully ionic power source $\uparrow$
}

\author{
Song Yi Yeon, $\dot{t}^{\mathrm{a}}$ Jeongse Yun, $\hat{\hbar}^{\mathrm{a}}$ Sun-heui Yoon, ${ }^{\mathrm{a}}$ Dahye Lee, ${ }^{a}$ Woohyuk Jang, ${ }^{\mathrm{a}}$ \\ Seok Hee Han, ${ }^{a}$ Chung Mu Kang ${ }^{b}$ and Taek Dong Chung (DD *ab
}

A novel pump-free miniaturized reverse electrodialysis (RED) system was designed to provide lasting power transduced from salinity gradients, named solid salt RED (sSRED), and this quasi-battery uses a solid salt instead of electrolyte solution for streamlined usage. It is portable, flexible, comparable in size to a universal serial bus flash drive, and easily activated with a small amount of water. It maintains a constant ionic concentration gradient through precipitation reactions between a pair of different salts. This precipitation-assisted solid salt RED (PsSRED) is an unprecedented ionic power source as it can generate steady electricity in the absence of a driving pump. The PsSRED was successfully coupled with bipolar electrode (BPE) microchip sensors which require stable ionic electricity and a polyelectrolyte ionic diode to realize a fully ionic circuit. It is envisioned that the range of application could be expanded to supply electromotive force to various devices through an ionic charge flow.

Received 4th July 2018

Accepted 27th August 2018

DOI: $10.1039 / \mathrm{c} 8 \mathrm{sc} 02954 \mathrm{~g}$

rsc.li/chemical-science

in the human body to perform various functions such as sensing, interfacing and drug delivery.

However, the ionic-electronic interfaces that apply elecBeing vital to biological activity, ionic systems form the basis of physiological processes in all living organisms. Through tight regulation of ion transport, key biological processes are governed. For instance, nerve signals are transmitted via potassium-sodium concentration gradients within nerve cells, and energy production is facilitated by a proton gradient which powers adenosine triphosphate (ATP) synthesis.

Inspired by biological systems, completely ionic circuits have been developed to understand and control the behavior of ions, and to communicate between electronic circuits and organisms. Since Bockris first reported on electrolytic junctions, ${ }^{1}$ various ionic devices such as ionic diodes, ${ }^{2,3}$ ionic transistors ${ }^{4,5}$ and ionic logic gates ${ }^{6-8}$ have been studied to precisely control the ionic species in the electrolyte. Based on the working principle of the aforementioned devices, a wide range of functional materials has been reported by researchers based on inorganic and organic materials. ${ }^{9,10}$ In line with these efforts, polyelectrolytes were proposed as an attempt to bridge the gap between solid electronic and ionic systems. These electronic components are flexible, light-weight, environmentally benign, and biocompatible, ${ }^{2}$ with the aim of mounting on or implanting

\footnotetext{
${ }^{a}$ Department of Chemistry, Seoul National University, Seoul, 08826, Republic of Korea. E-mail: tdchung@snu.ac.kr

${ }^{b}$ Advanced Institute of Convergence Technology, Suwon-si, Gyeonggi-do, 16229, Republic of Korea

$\dagger$ Electronic supplementary information (ESI) available: Supplementary figures. See DOI: $10.1039 / \mathrm{c} 8 \mathrm{sc} 02954 \mathrm{~g}$

$\ddagger$ These authors contributed equally to this work.
} trical energy to an ionic environment present certain concerns. Firstly, interfacial charge transfer, also known as the faradaic reaction, can potentially be unfavorable to the biosystem ${ }^{11-14}$ and for analysis. ${ }^{15}$ In addition, conventional power sources are not bio-compatible because they require toxic materials and are not flexible. Thus, further modification and careful design of power sources are required for them to be compatible with bio-electronic interfaces and disposable sensors. Therefore, a new concept of power source that generates an ionic flow without causing bio-compatibility issues is in demand. To this end, for instance, electronic batteries which could serve as alternatives have been suggested using different electrode materials and ionic cables soaked with various ions which generates a selective flux of $\mathrm{Li}^{+}, \mathrm{Na}^{+}, \mathrm{K}^{+}$, and $\mathrm{Ca}^{2+}$ ions. ${ }^{16}$

Towards this objective, we have focused on reverse electrodialysis (RED). Since the landmark proof-of-concept by Pattle in $1954,{ }^{17}$ RED has garnered widespread attention from researchers as a promising source of renewable energy which could harvest a large amount of electricity from a mixture of river and sea water. ${ }^{18-21}$ Moreover, RED is an ideal power source surrogate to couple with various ionic devices, since it can generate ionic electricity without the need for solid conductors such as metal electrodes. ${ }^{22}$ However, the necessity for a mechanical pump to maintain the power of RED is cumbersome, as ionic devices are usually incorporated within microchips. In our previous studies, we proved that miniaturized RED could drive a bipolar electrochemical sensor and 
an iontophoretic drug delivery device by solely ionic means. ${ }^{2324}$ However, rapid power decay in the absence of a pump and tedious injection of saline solutions to every chamber of the miniaturized RED have greatly hindered its practicality.

We, thus, wished to devise an ultimate miniaturized REDbased power source that could surmount the previous systems, named precipitation-assisted RED (PssRED, vide infra). If it could serve as an economical and convenient means to provide stable power over a longer duration of time without the aid of a pump, we believed that it could potentially be coupled with various electronic devices to achieve practicality. The operation of two completely ionic model devices, bipolar electrode (BPE) based sensing apparatus and a polyelectrolyte based ionic diode, is presented to highlight the utility of the newly developed PsSRED system. BPEs, being electrically disconnected electrodes, are hence well suited for miniaturized devices that are unwieldy for wiring. Therefore, bipolar electrode-electrochemiluminescence (BPE-ECL) sensing of many targets is intensively studied, ${ }^{25-28}$ leading to a surge in demand for smaller, simpler, and lighter power sources. ${ }^{29}$ Thus, here, we examine PssRED as a stable power source for the BPE sensing system by showcasing efficient quantification of important target analytes, benzyl viologen (BV), ${ }^{25,30}$ a member of the viologen family in which various enzymatic redox mediators are included, ${ }^{31,32}$ and $\mathrm{H}_{2} \mathrm{O}_{2},{ }^{33-35}$ a key indicator of cellular oxidation. ${ }^{36}$ The second model system, the polyelectrolyte ionic diode, allows ionic current to be rectified, and thus can model biological information processing. ${ }^{22,30}$ These ionic circuits could serve as a key component for the construction of a biomimetic device for "iontronic" functions. ${ }^{37}$ Such iontronic devices are envisioned to enable the merger of classical and bio-electronics by communicating between electronic devices and the biological system. We, therefore, demonstrate that PSSRED could serve as an efficient power source for controlling the ionic flow by manipulating the ionic distributions in the polyelectrolyte ionic diode. These two examples could confirm the suitability of PssRED as a durable power source for various devices that require an ionic power supply.

\section{Experimental}

\section{Reagents and apparatus}

All chemicals were used as received without further purification. Tris(2,2'-bipyridyl)dichlororuthenium(II) hexahydrate $\left(\mathrm{Ru}(\mathrm{bpy})_{3} \mathrm{Cl}_{2} \cdot 6 \mathrm{H}_{2} \mathrm{O}\right)$, tripropylamine (TPA), benzyl viologen, barium chloride, silver sulfate, diallyldimethylammonium chloride (DADMAC), 2-acrylamido-2-methyl-1-propanesulfonic acid (AMPSA), sodium chloride, potassium chloride, 2-hydroxy-4'-(2hydroxyethoxy)-2-methylpropiophenone, fluorescein sodium salt, 3-(trimethoxysilyl)propyl methacrylate, methanol, silver wire (dia. $0.5 \mathrm{~mm}$ ), platinum wire (dia. $0.5 \mathrm{~mm}$ ), and indium tin oxide coated glass (ITO, 8-12 $\Omega$ per square) were purchased from Sigma Aldrich (St. Louis, Missouri, U.S.A.). An Ag/AgCl reference electrode was purchased from BASi (West Lafayette, Indiana, U.S.A.). Hexamethyldisilazane (HMDS), ethanol, hydrogen peroxide (30\% aq.), sulfuric acid and acetone were obtained from J. T. Baker (Phillipsburg, New Jersey, U.S.A.). A photoresist (PR; AZ 4620) and developer (AZ 400K) were purchased from Merck (Kenilworth, NJ, U.S.A.). A TIN etchant (TE-100) was purchased from Transene Company (Danvers, MA, U.S.A.). An anion exchange membrane (Selemion AMV) and cation exchange membrane (CMV) were products of Asahi Glass Co., Ltd. (Tokyo, Japan). Waterproof double-sided tape (ACE CROSS SBX) was purchased from Koyo-kagaku Co., Ltd. (Soka, Japan) and further customized by Teratec (Daegu, Korea). Deionized water (18 $\mathrm{M} \Omega \mathrm{cm})$ was obtained from a NANO pure Diamond (Barnstead, New Hampshire, U.S.A). A potentiostat (Reference 600, Gamry/CHI604 and $\mathrm{CHI} 750, \mathrm{CH}$ Instrument), digital camera (Canon EOS 750D, ISO 6400), microscope (Nikon TE2000U) and PMT detector (H10722-20, Hamamatsu) were used for electrochemical and optical measurements.

\section{Preparation of miniaturized solid salt REDs}

Miniaturized solid salt REDs were prepared by stacking cation exchange membranes (CEM) and anion exchange membranes (AEM) alternatively in two parallel columns, as shown in Fig. 1a. Double-sided waterproof tape $\left(4.0 \times 2.0 \mathrm{~cm}^{2}, 250 \mu \mathrm{m}\right.$ per layer $)$ was used as the template. A CEM and an AEM were attached over each hole of the waterproof tape, respectively. The high concentration and the low concentration chambers alternated between layers. On the uppermost layer was placed threelayered waterproof tape with a rectangular chamber $(3.0 \times 1.0$ $\mathrm{cm}^{2}$ ) to connect the two columns. Salt powder was delivered using a laboratory spatula without precise measurement, but was sufficient to reach saturation, when the membranes were stacked layer-by layer.
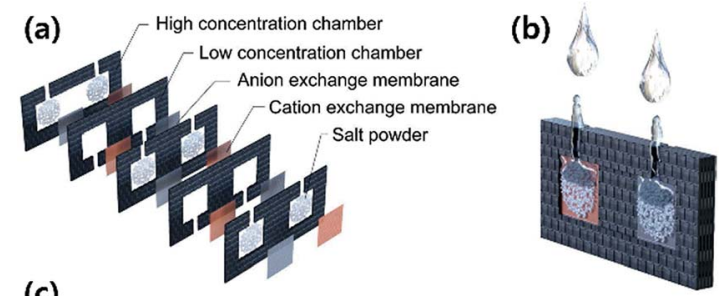

(c)

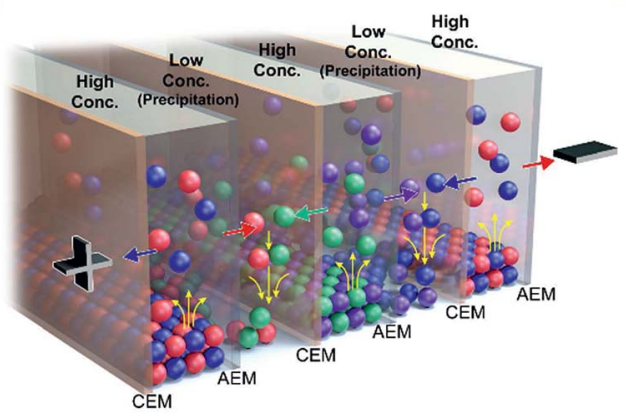

Fig. 1 (a) Layered structure of the SSRED consisted of alternating HC and LC chambers. The outermost membranes constitute the interface with the target devices. (b) Schematic diagram of SSRED activation. Entry of water through inlets and dissolution of solid salts in high concentration chambers activate the SSRED. (c) Working principles of PsSRED operation. 


\section{Characterization of the miniaturized solid salt REDs}

For operation, the ssREDs were immersed into deionized or tap water, pressed and released with fingers to fill each layer. The performance of miniaturized ssREDs was characterized by using a four-electrode system. Current flowing through the RED cell was galvanostatically driven by two platinum wires connected to a potentiostat (Reference 600, Gamry), while the potential was measured by using two $\mathrm{Ag} / \mathrm{AgCl}$ reference electrodes with a second potentiostat (CHI604, $\mathrm{CH}$ Instrument). Current was gradually increased from 0 to $200 \mu \mathrm{A}$ with a step size of $10 \mu \mathrm{A}$.

\section{Fabrication of an ITO electrode and BPE microchip}

The BPE microchip consisted of upper patterned glass and lower ITO-electrode-embedded glass. A glass slide was washed with piranha solution for $40 \mathrm{~min}$ and ITO-coated glass was washed with ethanol, acetone, and deionized water, successively and baked at $150{ }^{\circ} \mathrm{C}$ for $15 \mathrm{~min}$. HMDS was spin-coated on the glass surfaces and baked at $110{ }^{\circ} \mathrm{C}$ for $1.5 \mathrm{~min}$. Then, PR (AZ 4620) was spin-coated and baked at $100{ }^{\circ} \mathrm{C}$ for $1.5 \mathrm{~min}$. UV light (365 $\left.\mathrm{nm}\right)$ was illuminated on the PR layer through a mask with a channel pattern and BPE masked pattern. Then the PR pattern was developed using an AZ 400K developer and baked at $120^{\circ} \mathrm{C}$ for $15 \mathrm{~min}$. The upper glass was immersed in a buffered oxide etchant for $45 \mathrm{~min}$ and the lower ITO-coated glass was etched with TE-100. Four holes were drilled on the upper glass for solution reservoirs. Finally, the glasses were sonicated in acetone and were both submerged in $\mathrm{NH}_{4} \mathrm{OH}\left(30 \%\right.$ aq.) : $\mathrm{H}_{2} \mathrm{O}_{2}(30 \%$ aq.) : D.I. water $(2: 1: 2)$ solution at $180{ }^{\circ} \mathrm{C}$ for $30 \mathrm{~min}$. They were heated to $500{ }^{\circ} \mathrm{C}$ in a furnace (CRF-M15, Ceber, Korea) for $12 \mathrm{~h}$ for complete attachment.

\section{Electrochemical and optical analysis}

Cyclic voltammetry was performed by using a potentiostat (CHI750, CH Instruments). The three-electrode system consisted of ITO coated glass as the working electrode, Pt wire as the counter electrode, and $\mathrm{Ag} / \mathrm{AgCl}$ as the reference electrode.

The reporting channel on the anodic side was filled with $0.1 \mathrm{M}$ PBS (pH 6.9) containing $5 \mathrm{mM} \mathrm{Ru}(\mathrm{bpy})_{3} \mathrm{Cl}_{2} \cdot 6 \mathrm{H}_{2} \mathrm{O}$ and $25 \mathrm{mM}$ TPA. The detecting channel for BV reduction on the cathodic side was filled with $0.1 \mathrm{M}$ PBS ( $\mathrm{pH}$ 6.9) containing different concentrations ( 0 to $10 \mathrm{mM}$ ) of BV. For detection of $\mathrm{H}_{2} \mathrm{O}_{2}$, it was filled with $0.1 \mathrm{M}$ PBS ( $\mathrm{pH}$ 6.9) containing different concentrations ( 0 to $10 \mathrm{mM}$ ) of $\mathrm{H}_{2} \mathrm{O}_{2}$. Constant voltage was applied to the both ends by using either a potentiostat or a PSSRED. When a potentiostat was used, potential was applied through $\mathrm{Ag} / \mathrm{AgCl}$ driving electrodes (diameter: $0.5 \mathrm{~mm}$ ). Otherwise, an activated PSSRED was attached wirelessly to the BPE chip. For both cases, the ECL images were obtained in a dark room with an exposure time of $15 \mathrm{~s}$ using a digital camera (Canon EOS 750D, ISO 6400). The ECL intensities of the images were analyzed by using ImageJ software.

\section{Detection of ECL intensity by using a photomultiplier tube} detector

After filling $10 \mathrm{mM} \mathrm{H}_{2} \mathrm{O}_{2}$ and ECL solution into both microchannels of the BPE, a PSSRED and NSSRED are respectively attached. The resulting ECL intensities were measured with a microscope (Nikon TE2000U) connected to a PMT detector (H10722-20, Hamamatsu) for 10 minutes. The obtained signals were analyzed using a self-programmed LabVIEW (LabVIEW 2015, National Instruments) program.

\section{Fabrication of a polyelectrolyte ionic diode and measurement} of fluorescence

$\mathrm{X}$-shaped diode chips were prepared by following previously reported procedures with dimensions shown in Fig. S4c. $\dagger^{22}$ Fluorescein $(1.7 \mu \mathrm{M}$ in $0.1 \mathrm{M}$ aqueous $\mathrm{KCl}$ ) was loaded into both channels. A 30-Stack PsSRED was directly attached to the channel openings of the X-shaped diode chips as shown in Fig. 4a. The sign of bias was controlled by the direction of attachment. Fluorescence from an n-type polyelectrolyte gel was observed using a fluorescence microscope (TE2000U, Nikon, Japan).

\section{Results and discussion}

\section{Strategies and design for solid salt REDs}

The PSSRED was strategically designed as follows: first, we envisioned to replace saline solution with a solid salt in a high concentration (HC) chamber to avoid tedious injection processes (Fig. 1a). We believed that, with this RED named the solid salt RED (SSRED), the activation protocol could be simplified to a one-shot addition of a small quantity of water (Fig. 1b). Moreover, in terms of efficiency and economy, because the RED would stay dry prior to activation, it would be possible to be stored and distributed over longer time periods without any significant deterioration of performance. Secondly, ensuring that the ssRED maintains a prolonged steady state potential was of key importance. Therefore, to preserve low ionic concentrations in the LC chambers, we presented an unprecedented approach of introducing two different salts, which forms insoluble precipitates after ionic metathesis, alternatively into the HC chambers (Fig. 1c). This type of SSRED is named the precipitation-assisted SSRED (PssRED). Two kinds of ssREDs are presented in this paper: one using only $\mathrm{NaCl}$ (NssRED) and other using $\mathrm{BaCl}_{2}$ and $\mathrm{Ag}_{2} \mathrm{SO}_{4}$ (PssRED) (Fig. $\left.\mathrm{S} 1 \dagger\right)$. The ssREDs are small $(4.0 \times 2.0$ $\mathrm{cm}^{2}$, thickness $250 \mu \mathrm{m}$ per layer), comparable to a common universal serial bus (USB) flash drive and weigh only approximately $3 \mathrm{~g}$ (20 stack SSRED).

The theoretical verification of PssREDs is described as follows: two kinds of salts, $\mathrm{BaCl}_{2}$ and $\mathrm{Ag}_{2} \mathrm{SO}_{4}$, are placed alternatively in $\mathrm{HC}$ chambers, and then they are dissolved and ionized after the activation. Their solubility (maximum mass (g) of solute dissolvable in $100 \mathrm{~mL}$ of water) is 37 and 0.84 , respectively. ${ }^{38}$ The cations and anions in the HC chambers diffuse in opposite directions, forming barium sulfate $\left(\mathrm{BaSO}_{4}\right)$ and silver chloride $(\mathrm{AgCl})$ by ionic metathesis in the LC chambers. These salts are insoluble as solubility products in 
water are $1.1 \times 10^{-10}$ and $1.8 \times 10^{-10}$, respectively. ${ }^{39}$ Therefore, precipitation keeps the concentration of LC chambers low so that the salinity ratio of each ion between HC and LC chambers in an open circuit should be $1.64 \times 10^{5}, 2.81 \times 10^{5}$, $4.21 \times 10^{3}$ and $2.54 \times 10^{3}$, for $\mathrm{Ba}^{2+}, \mathrm{Cl}^{-}, \mathrm{Ag}^{+}$and $\mathrm{SO}_{4}{ }^{2-}$, respectively.

\section{Performance evaluation of ssREDs}

The performance evaluation of ssREDs as a power source is presented in Fig. 2. We obtained the normalized time-dependent decay of open circuit potentials (OCPs) to compare the durability of the NssRED and PsSRED. Reportedly, the ion selectivity of the membranes is imperfect so that counterion transport reduces the concentration difference between $\mathrm{HC}$ and LC chambers, resulting in the rapid degradation of OCPs. ${ }^{40}$ However, the rate of decay is significantly lowered when the precipitation reaction take place in the LC chamber: the OCP of the NSSRED rapidly decreases to $50 \%$ after $1500 \mathrm{~s}$ and completely diminishes after $5000 \mathrm{~s}$, while that of the PsSRED decreases only marginally, preserving $90 \%$ of the maximum OCP even after $19000 \mathrm{~s}$. This clearly shows that precipitation reactions suppress the concentration building up in the LC chamber as expected.

The output voltage is easily adjustable as shown in Fig. $\mathrm{S} 2 \dagger$ where the OCP exhibits a linear correlation with the number of stacks with a slope of about $85 \mathrm{mV}$ per stack, which is $85 \%$ of the calculated value. The charge that can be delivered by the PssRED would be proportional to the initial salt loading as shown in Fig. S3. $\dagger$ As demonstrated in the current-potential curve shown in Fig. $2 \mathrm{~b}$, the maximum power of a 20-stack PssRED is approximately $100 \mu \mathrm{W}$. Although this value is much lower than that required for devices that consume large electricity, it is enough to operate small devices like microfluidic ionic circuits.

\section{BPE sensing systems powered by ssREDs}

To see if PssREDs could successfully work as a new type of power source for low power devices, we conducted a series of experiments using an RED-powered BPE sensing platform (Fig. 3a). This bipolar system consists of microchannels and an indium tin oxide (ITO) electrode lying underneath. The detecting and reporting channels are separated to prevent mutual interference (Fig. S4a†). The ECL signal coupled with the

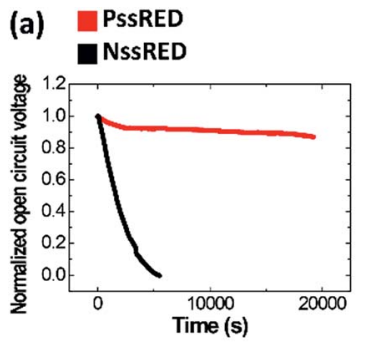

(b)

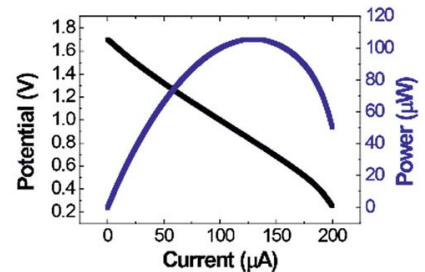

Fig. 2 (a) Normalized open circuit potential (OCP)-time curve of a 4stack PsSRED (red) and normalized OCP-time curve of the NsSRED (black). (b) Power (blue) and potential (black) curve of a 20-stack PsSRED versus operating current. (a)

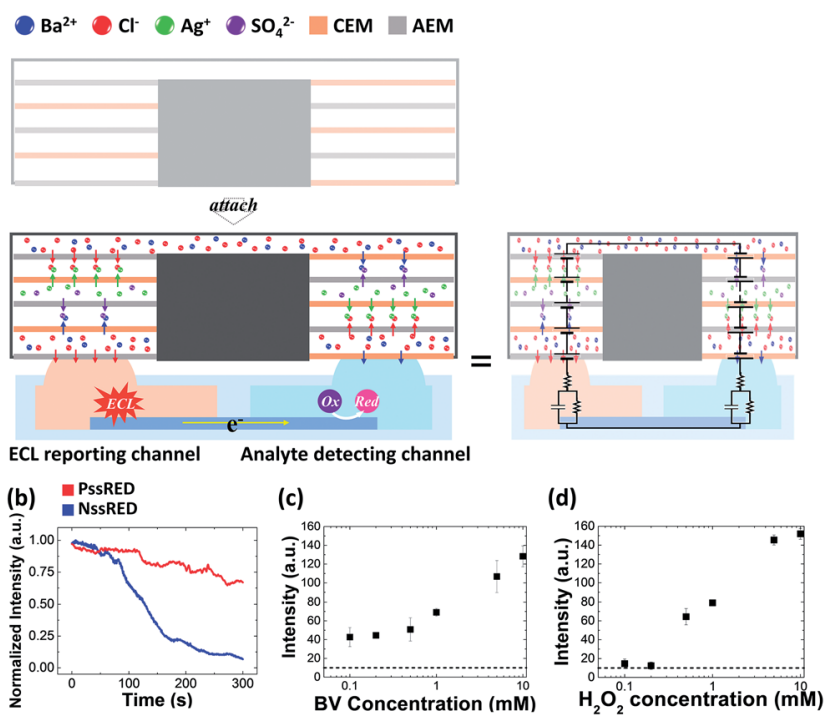

Fig. 3 (a) Schematic diagram (left) and equivalent circuit (right) of a RED powered BPE sensing chip. PssRED is placed on an ITO-BPE microchip without any metallic contact. (b) Normalized ECL intensity over time using a BPE chip powered by a PsSRED (red) and NssRED (blue). Analyte was $10 \mathrm{mM}$ solution of $\mathrm{H}_{2} \mathrm{O}_{2}$ in $\mathrm{PBS}$ (pH 6.9). ECL intensity versus the concentration of (c) BV and (d) $\mathrm{H}_{2} \mathrm{O}_{2}$ using a 30stack PsSRED powered BPE chip. Reporting channel: $5 \mathrm{mM} \mathrm{Ru}(\mathrm{bpy})_{3} \mathrm{Cl}_{2}$ and $25 \mathrm{mM}$ TPA. Detecting channel: $0.2-10 \mathrm{mM} \mathrm{BV}$ and $\mathrm{H}_{2} \mathrm{O}_{2}$. All the solutions were made in $\mathrm{pH} 6.9 \mathrm{PBS}$.

reduction of model analysts, benzyl viologen and hydrogen peroxide, could be detected either by using a digital camera or the naked eye.

Quantitative analysis was performed by using a 30-stack PssRED-powered BPE sensing platform (for the selection of the driving voltage, see Fig. S5 $\dagger$ for details). As soon as a direct contact between the BPE cell and the PssRED was made, ECL emission was observed at the anode of the BPE.

First, we investigated the durability of ECL intensity because persistent ECL emission is crucial for consistent detection. It was measured using a homemade ECL analyzer consisted of a microscope and a photomultiplier tube (PMT). The ECL emission powered by the PssRED was constant for the first $100 \mathrm{~s}$ and lasted for $300 \mathrm{~s}$ with intensity decreasing to merely $c a$. 70\% of its initial value, while the ECL from the NssRED-powered BPE started to diminish immediately, decreasing to $c a$. $70 \%$ of its initial value in $100 \mathrm{~s}$ (Fig. 3b). The slight decrease of PssREDpowered ECL emission is not due to the power drop of the PSSRED as the OCP of the PsSRED recovered fully after the measurement.

Next, calibration curves were constructed with a digital camera as the detector to take into account the actual environment where a high-end instrument is not affordable (Fig. 3c and d). ECL emission was observed as soon as the direct contact between the PssRED and BPE cell was made (Fig. S6 $\dagger$ ). The adsorption of $\mathrm{BV}$ to the ITO electrode resulted in higher background emission compared to that of $\mathrm{H}_{2} \mathrm{O}_{2}$ (Fig. S7 $\dagger$ ). Owing to its stability and durability, a single PssRED could run more than twenty BPE-ECL analyses once it is activated, even though each 
(a)

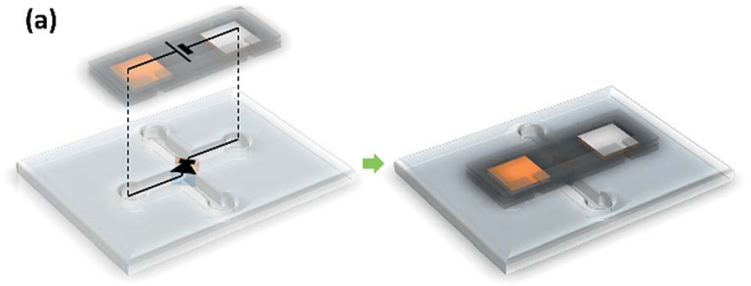

(b)
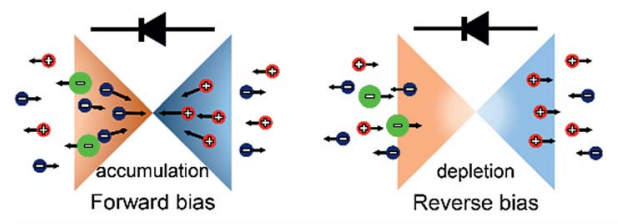

(c)

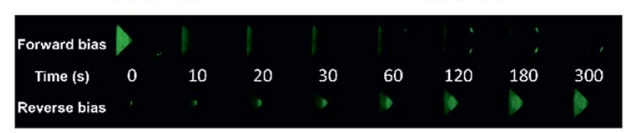

Fig. 4 (a) Equivalent circuit and schematic diagram of a polyelectrolyte ionic diode powered by a PsSRED. (b) Working principle of the polyelectrolyte ionic diode system under each bias. (c) Temporal fluorescence images of the $n$-type polyelectrolyte gel (forward bias: upper row and reverse bias: lower row).

measurement took approximately $15 \mathrm{~s}$. The whole calibration curve, hence, could be obtained with a single PssRED. Overall, the PSSRED powered BPE sensing platform showed analytical performance comparable to that powered by a potentiostat (Fig. S8 $\dagger$ ), corroborating that the PssRED is an effective power source for small sensing devices.

\section{Polyelectrolyte ionic diodes powered by SSREDs}

Finally, to confirm the possibilities of operating an ionic circuit with the PssRED system, the polyelectrolyte ionic diode previously reported by our group ${ }^{7,22}$ was driven with a 30-stack PssRED. Fig. 4a depicts the equivalent circuit and the direct attachment of the PsSRED to the X-shaped polyelectrolyte ionic diode. In the polyelectrolyte ionic diode system shown in Fig. 4 b, the counterions build up in the junction under forward bias, but dissipate to the bulk solution under reverse bias. Since the mobility of $\mathrm{Cl}^{-}$is much larger than that of fluorescein (shown as green anions in Fig. 4b), it would push fluorescein against the electric field. Thus, under forward bias, fluorescein is driven out of the gel due to the influx of $\mathrm{Cl}^{-}$ions, and vice versa under reverse bias. Fig. 4c shows the temporal fluorescence measurements of the X-shaped diodes containing fluorescein solutions as the electrolyte. Fluorescence diminished over time under forward bias and intensified under reverse bias, confirming that the fully ionic circuit composed of ionic diodes and an ionic power source is successfully operating. This serves as an example to actually prove the capacity of PssREDs in driving ion-based systems, which eventually allow them to expand to other biomimetic devices.

\section{Conclusions}

In this work, we proposed a new concept of battery that functions without any metallic components or other solid conducting materials. It is based on the working principle of RED where the salinity gradient is transduced into electricity. The use of a pump, a necessary component to maintain constant electricity but a critical drawback for miniaturization, is removed by employing a pair of dry salts. When an RED contains a dry salt in HC chambers, it can be easily activated by simply immersing in water. The stability and durability of ssREDs were remarkably improved by using a pair of soluble salts that can form insoluble salts by exchanging their counterions (PssRED). Precipitation reactions in LC chambers maintain low concentrations so that electromotive force is maintained stable for several hours. The PssRED can supply consistent power to run small devices, demonstrated through driving a bipolar-ECL sensor apparatus and a polyelectrolyte ionic diode. Furthermore, the operating voltage could be customized for target usage by adjusting the number of stacks. We believe that the PssRED exhibits exceptional prospects for applications to a wide range of ion-based platforms such as biointerfacing and drug delivery, and further investigation is ongoing in our laboratory.

\section{Conflicts of interest}

There are no conflicts to declare.

\section{Acknowledgements}

This research was supported by the Nano Material Technology Development Program through the National Research Foundation of Korea (NRF) funded by the Ministry of Science, ICT and Future Planning (2011-0030268).

\section{References}

1 B. Lovrecek, A. Despic and J. O. M. Bockris, J. Phys. Chem., 1959, 63, 750.

2 O. J. Cayre, S. T. Chang and O. D. Velev, J. Am. Chem. Soc., 2007, 129, 10801.

3 E. O. Gabrielsson, P. Janson, K. Tybrandt, D. T. Simon and M. Berggren, Adv. Mater., 2014, 26, 5143.

4 K. Tybrandt, E. O. Gabrielsson and M. Berggren, J. Am. Chem. Soc., 2011, 133, 10141.

5 K. Tybrandt, K. C. Larsson, A. Richter-Dahlfors and M. Berggren, Proc. Natl. Acad. Sci. U. S. A., 2010, 107, 9929.

$6 \mathrm{~K}$. Tybrandt, R. Forchheimer and M. Berggren, Nat. Commun., 2012, 3, 871.

7 J.-H. Han, K. B. Kim, H. C. Kim and T. D. Chung, Angew. Chem., Int. Ed., 2009, 48, 3830.

8 H. Sun, M. Vagin, S. Wang, X. Crispin, R. Forchheimer, M. Berggren and S. Fabiano, Adv. Mater., 2018, 30, 1704916.

9 P. Ramirez, J. Cervera, M. Ali, W. Ensinger and S. Mafe, ChemElectroChem, 2014, 1, 698-705.

10 C. J. Wan, L. Q. Zhu, J. M. Zhou, Y. Shi and Q. Wan, Nanoscale, 2013, 5, 10194.

11 Y. Li, M. Wu, D. Zhao, Z. Wei, W. Zhong, X. Wang, Z. Liang and Z. Li, Sci. Rep., 2016, 5, 17817. 
12 M. Grys, Z. Madeja and W. Korohoda, Cell. Mol. Biol. Lett., 2017, 22, 1.

13 B. Taji, S. Shirmohammadi, V. Groza and I. Batkin, IEEE Trans. Instrum. Meas., 2014, 63, 1412.

14 P. F. Meyer, P. D. Gadsby, D. Van Sickle, W. E. Schoenlein, K. S. Foster and G. P. Graber, Med. Biol. Eng. Comput., 2005, 43, 225.

15 S. E. Fosdick, K. N. Knust, K. Scida and R. M. Crooks, Angew. Chem., Int. Ed., 2013, 52, 10438.

16 C. Wang, K. K. Fu, J. Dai, S. D. Lacey, Y. Yao, G. Pastel, L. Xu, J. Zhang and L. Hu, Nat. Commun., 2017, 8, 15609.

17 R. E. Pattle, Nature, 1954, 174, 660.

18 B. E. Logan and M. Elimelech, Nature, 2012, 488, 313.

19 M. Tedesco, A. Cipollina, A. Tamburini and G. Micale, J. Membr. Sci., 2017, 522, 226.

20 J. G. Hong, B. Zhang, S. Glabman, N. Uzal, X. Dou, H. Zhang, X. Wei and Y. Chen, J. Membr. Sci., 2015, 486, 71.

21 X. Tong, B. Zhang and Y. Chen, J. Membr. Sci., 2016, 516, 162.

22 S. H. Han, S.-R. Kwon, S. Baek and T.-D. Chung, Sci. Rep., 2017, 7, 14068.

23 S.-R. Kwon, S. H. Nam, C. Y. Park, S. Baek, J. Jang, X. Che, S. H. Kwak, Y.-R. Choi, N.-R. Park, J.-Y. Choi, Y. Lee and T. D. Chung, Adv. Funct. Mater., 2018, 1705952.

24 S. Baek, S.-R. Kwon, S. Y. Yeon, S.-H. Yoon, C. M. Kang, S. H. Han, D. Lee and T. D. Chung, Anal. Chem., 2018, 90, 4749.

25 W. Zhan, J. Alvarez and R. M. Crooks, Anal. Chem., 2003, 75, 313.
26 B.-Y. Chang, K.-F. Chow, J. A. Crooks, F. Mavré and R. M. Crooks, Analyst, 2012, 137, 2827.

27 W.-X. Lu, N. Bao and S.-N. Ding, RSC Adv., 2016, 6, 2538825392.

28 M.-S. Wu, D.-J. Yuan, J.-J. Xu and H.-Y. Chen, Chem. Sci., 2013, 4, 1182.

29 F. Mavré, R. K. Anand, D. R. Laws, K.-F. Chow, B.-Y. Chang, J. A. Crooks and R. M. Crooks, Anal. Chem., 2010, 82, 8766.

30 W. Zhan, J. Alvarez and R. M. Crooks, J. Am. Chem. Soc., 2002, 124, 13265.

31 A. L. de Lacey, M. T. Bes, C. Gómez-Moreno and V. M. Fernández, J. Electroanal. Chem., 1995, 390, 69.

32 C. L. Bird and A. T. Kuhn, Chem. Soc. Rev., 1981, 10, 49.

33 N. Nasirizadeh, Z. Shekari, A. Nazari and M. Tabatabaee, J. Food Drug Anal., 2016, 24, 72.

34 A. Noorbakhsh, M. Khakpoor, M. Rafieniya, E. Sharifi and M. Mehrasa, Electroanalysis, 2017, 29, 1113.

35 L. Wang, Electrochem. Commun., 2004, 6, 225.

36 S. Z. Bas, C. Cummins, D. Borah, M. Ozmen and M. A. Morris, Anal. Chem., 2018, 90, 1122.

37 H. Chun and T. D. Chung, Annu. Rev. Anal. Chem., 2015, 8, 441.

38 D. R. Lide, CRC Handbook of Chemistry and Physics, CRC Press, Boca Raton, FL, USA, 2003.

39 A. E. Martell and R. M. Smith, Critical Stability Constants. 4. Inorganic Complexes, Plenum Press, New York, NY, USA, 1976.

40 T. Sata, J. Membr. Sci., 1994, 93, 117. 